\title{
COMMUNITY AWARENESS ABOUT LEPROSY IN A DISTRICT OF NEPAL
}

\author{
Agrawal S*, Jha N*, Agarwalla A*, Agrawal C S*, Niroula S*
}

\section{ABSTRACT}

Nepal is one of the countries with high prevalence of leprosy. In general, the prevalence of the disease is relatively higher in the plains than in the hills. We analyzed the knowledge of leprosy in the community by a structured questionnaires study. The sources of information from where they acquired the knowledge were also studied. This study was done in Sunsari district of Nepal on National Immunization Day program. Out of 1647 respondents, $60 \%$ were female. The majority (74\%) was between $20-40$ years of the age group. Agricultural workers constituted 58\%. One third of the respondents were illiterates. Approximately $60 \%$ of the respondents knew the cause and symptoms of leprosy. "Leprosy is curable" was felt by $81.8 \%$. Although $75 \%$ of the respondents knew that drugs for leprosy were available free of cost, the goal of elimination was understood by only $51 \%$ of respondents. The younger individuals had better knowledge of the cause and availability of the free treatment of leprosy. The females had equal knowledge about cure, but had less knowledge of the cause, symptoms, availability of the free treatment and elimination goal. Illiterates, laborers and unemployed had significantly less knowledge of leprosy. There was no difference seen between different levels of education in literate groups. Radio was the single most effective means of providing information, education and communications about leprosy. A good knowledge of leprosy inculcates a positive attitude towards leprosy. In general this may result in better case reporting and regularity of treatment as well as fewer deformities.

\section{Key Words: Leprosy, Community Awareness.}

\section{INTRODUCTION}

Leprosy can cause severe deformities if left untreated or if the therapy is not instituted in time. It has also severe emotional and social effects on the individual's life. The fear of social isolation makes the sufferers reluctant to seek medical advice in the early stages of the disease or if the patient is suspicious of the diagnosis. The superstitions and myths attached to leprosy are presumed to be based on ignorance and misconceptions about the disease. This would disappear if the community were 'educated' with scientific information and knowledge about the disease. ${ }^{1}$
It is now widely recognized that health education is an essential component of leprosy control. Effective health education is important to encourage people to seek earlier treatment; to educate them about the disease and to create public support for the patients. ${ }^{2}$ The prevalence of leprosy has been reduced considerably in many endemic countries with the introduction of Multi-Drug Therapy (MDT). It is still a major public health problem in Nepal. Out of 75 districts in Nepal, 27 have a prevalence rate of $4.26 / 10,000$. In general, the prevalence of the disease is relatively higher in the plains compared to the hills. Many International Non-Government Organizations (INGO) and Non-Government Organizations (NGO) are

* B.P. Koirala Institute of Health Sciences, Dharan, Nepal.

Address for correspondence : Dr. Sudha Agrawal

Department of Dermatology

B.P. Koirala Institute of Health Sciences, Dharan, Nepal.

Email: sudha92@yahoo.com 
actively involved with the Leprosy Control Division, the Department of Health Services, HMG in Nepal to achieve the global elimination of leprosy. ${ }^{3}$

The present study was carried out to know the knowledge of leprosy in a community. The sources of information from where they acquired the knowledge were also studied.

Several studies ${ }^{4-9}$ have been conducted on community knowledge about leprosy or the effectiveness of health education in India, Burma and Bangladesh. Even though, Nepal is having a similar type of geography and culture, the educational schemes have to be adjusted depending upon the requirement of the local populations. These should be well planned to suit the particular population and the effectiveness of the education should be checked at intervals. To the best of our knowledge and literature search, a very few studies ${ }^{2,10}$ have been conducted from Nepal about the knowledge of leprosy in the community. Therefore, this study was undertaken in the Sunsari district of Nepal.

\section{MATERIALS AND METHODS}

The study was carried out during the National Immunization day (NID) and was conducted in the 200 -vaccination post of the 50 village development committees in the rural areas of Sunsari District, Nepal. Sunsari district lies in the plains of the eastern region in the Koshi Zone. It has a varied topography, ranging from 152 meters to 918 meters above sea level and consisting of terai $(80 \%)$ and middle hill $(20 \%)$. It has an area of $1257 \mathrm{~km}^{2}$ and a population of 591,430 with a density of 470.4 people per $\mathrm{km}^{2} .{ }^{11}$

A structured questionnaire was developed and the questionnaires were distributed to each vaccination post through the district health office one day before the NID. The NID was utilized for this study so that it was possible to collect the data in a day without many difficulties and with the same resources.
The questionnaire included the age, sex, education and occupation of the respondents as well as their knowledge about leprosy, leprosy elimination program and their source of information. The knowledge was assessed by including the questions on the cause, symptoms, availability of free treatment and whether the condition is curable.

The questionnaire was in Nepali language and contained guidelines for the health workers. To minimize bias, each health worker was asked to start the interview at 10AM. The health workers were asked to complete all questionnaires sequentially interviewing ten consecutive people and returning the same to the district health office and subsequently to the investigators. The results were analyzed using EPI Info software version 5.

\section{RESULTS}

Out of 2000 questionnaires, 1647 were received from the district health office. The response rate was $82.3 \%$. The respondents were predominantly females $(60 \%)$ and about $74 \%$ were in the age group of $20-40$ years. A total of $31.6 \%$ of the respondents were illiterate; of the $68.4 \%$ that could read, $28.3 \%$ were barely literate (could read and write but had no formal schooling). Regarding occupation of the respondents, agricultural workers constituted $58 \%$, followed by laborers (16.6\%), businessmen (6\%), students $(5.5 \%)$, Government service employees $(5 \%)$, private service employees $(4.8 \%)$, unemployed (3.3\%) and housewives (1\%). It is seen that $53 \%$ of the respondents knew 'germs' as the cause of Leprosy, followed by impurities of blood (19.8\%), curse from God $(16.9 \%)$ and others $(10.3 \%) .981$ respondents $(60 \%)$ enumerated the symptoms of leprosy. The key words " whitish patch" and "sensation loss" were mentioned by $76.5 \%$ and $60 \%$ respectively as the earliest symptoms. A few respondents felt wounds $(25 \%)$, tingling sensations $(21.2 \%)$, and deformities $(4.1 \%)$ were the first symptoms. "Leprosy is curable" was felt by $81.8 \%$. However, $75 \%$ of the respondents had knowledge about drugs for leprosy available free of the cost, the goal of elimination by 2003 was understood by only $51 \%$ of the respondents. 